\title{
A TENDENCY OF HIGHER PROPORTIONS OF WOMEN THAT HAVE MISCARRIAGES BEFORE RHEUMATOID ARTHRITIS DIAGNOSIS, A BRAZILIAN COHORT
}

Letícia Assad Maia Sandoval (Universidade de Brasília, Brasília, DF, Brasil), Lidiana Bandeira Santana (Hospital Universitário de Brasília, Brasília, DF, Brasil), Talita Yokoy Souza (Hospital Universitário de Brasília, Brasília, DF, Brasil), Licia Maria Henrique Mota (Universidade de Brasília, Brasília, DF, Brasil), Luciana Ansaneli Naves (Universidade de Brasília, Brasília, DF, Brasil)

\section{BACKGROUND}

Rheumatoid arthritis (RA) is a chronic inflammatory disease associated with sub fertility. There has been reported a multifactorial etiology for sub fertility. In addition, there is an association of RA with poorer maternal fetal prognosis.

\section{MATERIALS AND METHODS}

Patients included had RA diagnosis by the American college of rheumatologists criteria. We questioned the reproductive life and maternal fetal record of 72 women.

\section{RESULTS}

The mean age was $53,7+15,42$ years and average age of onset of $R A$ was $43,71+14,93$ years. The average menarche age was 13,49 years, $54,9 \%$ of the patients older than 45 years were at menopause and haven't had hysterectomy surgery. 63 patients got pregnant and average time to conceive was 5,11 months. Total of 248 gestations were reported; patient's had an average of 3,44 pregnancies; 10 gestations were in a clinical scenario of RA; one gestation ended in an intentional miscarriage; $8,02 \%$ of all gestations that happened before RA diagnosis terminated in spontaneous miscarriages (SM) and $18,06 \%$ of the women evalluated had at least one SM before RA diagnosis. Two of those ten gestations concomitant with RA were followed by arthralgia; one had a preterm labor; others had a prolonged labor and one had preeclampsia and two ended in SM. Nine women didn't have gestations, mean age was $44+21,84$ years.

\section{CONCLUSION}

Average age of menarche and proportion of people older than 45 years at menopause at the brazilian national health research (NHR) of 2013 is 13 and 69,8\%, respectively. Most of the women have had children before the RA diagnosis. There were few pregnancies that occurs concomitant with RA, restricting conclusions regarding maternal and fetal prognosis. Interestingly, higher rates of women have had a SM before RA diagnosis (18,06\%) when compared to Brazilian NHR of 2013 witch reports 15,2\% proportion of women between 19 and 49 years had at least one SM. There are some limitations in these comparisons, because the age evaluated and the year of the research aren't the same. Nevertheless, this tendency SM before clinical manifestation are also reported in others autoimmune diseases, such as Hashimoto. We hypothesis an association between telomeres length and miscarriages. Since RA is associated with shorter telomeres, such as Hashimoto, witch also has a high rate of SM. We emphasize the necessity for further researchers to better evaluate this propensity of SM before clinical manifestation of autoimmune diseases. 